\title{
Surveying Iranian Authorities' Approach Toward Cyberspace Based on the Three Dimensions of Power by Steven Lukes
}

\author{
Majid Malekan \\ Seyyed Javad Emamjomeh Zadeh \\ Hossein Masoudnia \\ Ali Alihosseini \\ Department of Political Sciences, The University of Isfahan, Isfahan, Iran \\ majidmalekan@yahoo.com, javademam@yahoo.com, hmass2005@yahoo.com,ali.alihosseini@gmail.com
}

Doi:10.5901/mjss.2016.v7n3s1p62

\begin{abstract}
Cyberspace has had a significance effect on the relationship between governments and their citizens. Due to having different networks and its protest-like nature, the cyberspace challenges the power of governments, national governments and their sovereignty; thus governments try to have as much control as possible over the cyberspace in order to manage it toward their own benefits. Based on this, usage, partnership and policy making in the cyberspace is significantly affected by the approach and attitude of authorities in the society. The current research has used Steven Lukes's model of three dimensions of power as its theoretical framework to explain the relationship between cyberspace and political power in Iran; and it has analyzed the Iranian authorities' attitude and approach toward the cyberspace and it surveys that which of these approaches and attitudes are consistent with Steven Lukes's model. Research findings indicate that most approaches have had a non-optimistic attitude toward the cyberspace and only one approach has had an optimistic attitude toward the cyberspace. Also in Lukes's model, current approach of formal political power in Iran tends toward a two-dimensional approach and currently this policy is pursuing.
\end{abstract}

Keywords: Cyberspace, Political Power, Iranian Authorities, Steven Lukes.

\section{Introduction}

The current research main issue is surveying the Iranian authorities' approach and attitude toward cyberspace and its mechanisms. Regarding the cyberspace we encounter many different attitudes. Technological approach considers components such as hardware, software, quality and quantity of data transfer; whereas ,psychological approach analyzes components such as mentality, human and computer memes, identity and personality. Sociological approach considers online communities, social networks and social effects of human-computer interactions. Moreover, with emphasizing on cultural aspects and studying human behavior, anthropologists consider cyberspace as the product of interactions within the cultural system. But also there are other approaches such as political approach. In this framework explaining the relationship between cyberspace and the concept of power and especially imposing power are focused.

In cyberspace, evidences of power and the reaction of power applications are found. The first aspect of power is an actor's ability to force others to do things unlike their interests or their main strategies. Arresting dissident bloggers in 2009 in China is one of these events. Also an individual or organization may try to convince others to change their behavior.

The second aspect of power in cyberspace is developing or determining a plan in which an actor ignores the strategies of other actors and eliminate their upcoming options. Political censorship and imposing filtering in many countries around the world could be included in this framework.

The third aspect of political power in cyberspace is that an actor forms the main interests of other actors so that some strategies do not have the time to be emerged. Governments could also use advertisement for illegitimating some particular ideas.

Cyberspace significantly affects the relationship between governments and their citizens. The effect of internet on public sector policy is a dialectical effect: which means in one hand it decreases the individuals' dependence on traditional power references and it increases the citizens' power for making a wiser decision against these references and 
on the other hand this trend continues within the framework of the same patterns of dominating cultural system and power inequality.

Cyberspace and internet network challenges the power of governments, national governments and their sovereignty; this feature of cyberspace helps grow and promote anti-government forces, decreases the acceptability of governments by citizens, increases the power of anti-government forces in citizenship scale and global scale and generally it leads to threatening, weakening, destroying and substituting governments, national governments and their values and replaces rival forces and also decreases their authority.

Thus, the governments try to cope with this challenge and decrease or eliminate these threats against themselves. Sometimes they interpret the threats against themselves as threats against the national security and use it against their enemies and rivals; therefore, governments and the anti-government forces (whether with national scale or with global scale) compete with each other in cyberspace and challenge each other.

Along with this, determining the relationship between cyberspace and political power is very important in any society. For understanding this relationship it is possible to survey the approach and attitude of political power and its users toward the cyberspace in one hand and also it is possible to focus on the users' interactions in cyberspace and their attitude toward the concept of political power application. In this research, some indicators and components are extracted based on Lukes's theory and the viewpoints of political, cultural, judicial and military authorities on the cyberspace in this framework are analyzed. To address the issue, qualitative content analysis has been used; because there is a need for choosing samples and specific population in order to determine the type of relationship and the best data analysis method based on the existing statistical population is qualitative content analysis. This is because it is tried not to have any pre-assumptions of samples' comments, to have exploratory final data analysis and so that previous judgements do not have an effect on research procedure.

The most important objectives of this research are as follows:

1- Investigate the approach and views of the Iranian authorities toward cyberspace

2- Analysis of the imposing of political power in cyberspace and its consequences

3- Explanation of accepted pattern by political system in relation to cyberspace

4- Matching the agreed pattern with three dimensions of power by Steven Lukes

\section{Theoretical Framework}

In the current research Steven Lukes's theory of power is used for surveying the Iranian authorities' approach toward cyberspace. Lukes believes that in analyzing political power three different aspects of it could be displayed. Each one of these faces or dimensions considers a special mechanism for power application including: 1- one dimensional view which is pluralist and people such Dahl support this view. In this approach it is tried to study the specific works and results in order to designate individuals who have the main role in decision making and they can choose a suggestion among the proposed suggestions and make others accept those suggestions (Dahl, 1957: 15). Pluralists emphasize on objectivity of interests and they are against any claims saying that interests could be unclear or invisible and/or more importantly people could make mistakes regarding their interests or they may be unaware (Lukes, 1996: 17-20). If we consider one dimension for power then media is a tool that could be used by all individuals and groups for expressing their viewpoints; in such conditions, there is a market full of different media, messages, receivers and senders. Senders could transmit their messages through different media and receivers could choose from different messages based on their own interests. Although practically some groups have more access to media and have more facilities for sending their messages, this does not mean monopoly and other groups could also compete in this market and could change the situation in case of support of the audience. 2- Two-dimensional view: people such as Bachrach and Baratz are the representatives of this view. This is stated that the pluralists' analysis emphasizes on the exterior and public appearance of political power, whereas, a more complete analysis surveys two dimensions of decision making and non-decision making (Bachrach \& Baratz, 1962: 52). In fact, the second face or personal face of power could be observed in hidden elimination of individuals' or special groups' interests (Hindes, 2001: 20). These two theorists believe that onedimensional view of power has ignored the non-decision making matter which means the power to control subjects that are included in the political agenda and especially it has ignored eliminating some subjects from the political agenda (Bachrach \& Baratz, 1970: 7). In two-dimensional view, media is considered as a tool in the hands of powerful groups of the society by which they selectively broadcast their messages. In this view, media reports news and reports demanded by that powerful group. In such condition every message and piece of news threatening the benefits of sovereignty are censored and are not allowed to be broadcasted. 3- Three-dimensional view of power. Lukes criticizes one-dimensional 
and two-dimensional views and proposes the three-dimensional view. According to him power also acts in a third dimension and he calls it real benefits. Lukes distinguishes between intellectual benefits (what individuals think they want) and real benefits (what individuals may have wanted if they could have experienced the results and/or if they could have become aware of the alternative methods). Lukes's radical view states that human beings' demands may be the product of a system acting against their real benefits; thus detecting norms and institutionalized methods based on which intellectual benefits are replaced by real benefits shows the real face of third face of power. If we consider power as a three dimensional thing then media is a tool in the hands of powerful groups by which they institutionalize their values in the mind of people and convince the audience to voluntarily respect these values. In other words, instead of showing the reality and real benefits to the audience, media distort and manipulate their minds. According to this view, the main function of media is education and internalizing the lifestyle determined by authorities.

In this research, according to Lukes's theory, it is tried to determine that what view is chosen by the political power as its strategy to interact with the cyberspace. For evaluating this, a research model is considered based on which the indicators and models of political power application are categorized and then at the next stage these components and indicators are surveyed. In this framework the comments of senior authorities on Iranian cyberspace are analyzed (content analysis).

Power application methods in cyberspace by the government, features of desirable cyberspace policy, filtering or anti-filtering, attitude toward the user's freedom in cyberspace, authorities' attitude toward the cyberspace in terms of threats and opportunities and government's efforts for promoting thoughts and persuasion are components and indicators based on three dimensions of power by Lukes and they could be included in this theory and analyzed.

\section{Methodology}

Qualitative content analysis has been used for surveying the Iranian authorities' attitude toward cyberspace; because there is a need for choosing samples and specific population in order to determine the type of relationship and the best data analysis method based on the existing statistical population is qualitative content analysis. This is because it is tried not to have any pre-assumptions of samples' comments, to have exploratory final data analysis and so that previous judgements do not have an effect on research procedure.

Qualitative content analysis is one of the research useful qualitative techniques. Qualitative content analysis along with methods such as ethnography, grounded theory, phenomenology and historical research are some of the methods of content data analysis. Since late $20^{\text {th }}$ century when resistance against face analysis without paying attention to hidden content was raised, qualitative approaches in content analysis were developed (Mayring, 2000: 3).

Up to now different procedures and approaches have been proposed for qualitative content analysis. Hsieh and Shannon (2005) have shown three different qualitative content analysis approaches: Conventional content analysis, Directed content analysis and Summative content analysis. Conventional content analysis is usually used with study design and its aim is explaining a phenomenon. This method is useful when the theory or literature review about a phenomenon is limited. In this approach researchers stay away from pre-conceived concepts and let their concepts and titles be branched from data. Meyring has called this approach as inductive development. Directed content analysis has a structural process and its aim is conceptual validation of a theoretical framework or theory. In this approach the researchers use a theory or literature and start the procedure by determining concepts or key factors as the innovative coding variables. Summative content analysis starts with detecting and counting the words or specific content for understanding the content application of word or content (Hsieh \& Shannon, 2005: 1281-1284).

Based on the categorization provided by Hsieh \& Shannon, the current article approach is using conventional content analysis because our aim is explaining the content dimensions of authorities' comments and since there are no theories or literature review in this regard, it is better to let contents and titles be branched from data.

The current article main question in content analysis is that what kinds of theories discussed by Lukes about the dimensions of power include the authorities' approach regarding the cyberspace?

\section{Statistical Population \& Sample Selection}

The article case study includes the authorities' comments regarding internet and cyberspace. In this framework, all comments of senior authorities regarding internet and cyberspace are analyzed from 23/09/2009 to 22/09/2015 (5 years). The sampling method is purposive sampling and the ending time for sampling is when the contents are theoretically satiated and there are no new data during collection and further data analysis. The current research analysis unit is content. 
20 political figures including the Supreme Leader, leaders of mid-day congregational Friday prayers, authorities of the executive branch, legislature, the judiciary and military and police officials are chosen and their thoughts and comments about internet and cyberspace are analyzed. Surveying the comments of authorities, one of the important barriers of this research was choosing figures that firstly had a highly position and secondly they were selected by the authorities and thirdly had completely discussed their ideas. Based on this many important political figures that have not clearly and fully explained their ideas about internet and cyberspace (such as the president and former ministers) were eliminated from the analysis.

\section{Introduction to Analyzed Sample}

In this research, 20 political, cultural, and military figures are chosen and their names along with their positions are brought:

1- Ayatollah Seyed Ali Khamenei, The supreme leader

2- Ayatollah Ahmad Khatami, current leader of mid-day congregational Friday prayers in Tehran

3- Ayatollah Ahmad Alam Al-Hoda, leader of mid-day congregational Friday prayers in Mashhad

4- Ayatollah Ibrahim Amini, leader of mid-day congregational Friday prayers in Qom

5- Hojat Al-Islam Dr. Hassan Rouhani, president of the $11^{\text {th }}$ government

6- Ali Jannati, minister of culture of the $11^{\text {th }}$ government

7- Mahmoud Vaezi, minister of communications of the $11^{\text {th }}$ government

8- Hojat Al-Islam Seyyed Mahmoud Alavi, minister of intelligence of the $11^{\text {th }}$ government

9- Hojat Al-Islam Heydar Moslehi, minister of intelligence of the $10^{\text {th }}$ government

10- Mohammad Hassan Entezari, former secretary of the supreme council of cyberspace

11- Ayatollah Sadegh Amoli Larijani, head of the judiciary

12- Hojat Al-Islam Gholam Hossein Mohseni Ejei, the deputy and spokesman of the Judiciary

13- Hojat Al-Islam Abd Al-Reza Tarazi, special cybercrime judge

14- Dr. Gholam Ali Hadad Adel, member of Islamic consultative assembly

15- Hojat Al-Islam Ahmad Salek, chairman of the Cultural Commission of Islamic consultative assembly

16- Hojat Al-Islam Nasr Allah Pejmanfar, member of Cultural Commission of Islamic consultative assembly

17- Commander in chief Ismaeil Ahmadi Moghadam, former chief of police

18- Commander in chief Mohammadreza Naghadi, Basij commander

19- Commander in chief Hossein Nejat, cultural deputy of Army of the Guardians of the Islamic Revolution

20- Rouh Allah Nowbakht, cultural deputy of Mohammad the prophet of Allah Army

\section{The Main Axes of Analysis of Authorities' Approaches}

Comments of 20 top figures are categorized within 7 axes and analyzed:

\subsection{Inevitability and the necessity of using cyberspace}

One of the important issues in evaluating the authorities' view about the internet and cyberspace is inevitability and necessity of using it; in the Castells's network society theory it is also stated and it is considered as one of the indicators being analyzed in the research. In fact, internet and cyberspace have entered Iranian community as a new technology and tools and authorities' view regarding the necessity of using it and the reason to this necessity is an issue that is significantly indicates the attitude and approach to decision-making and planning regarding this communicational tool.

The inevitability and necessity of using cyberspace is reflected in views of 11 surveyed figures. Lack of pointing out to this issue is also either because of disbelief in the necessity or caused by sampling problems and requirements of the surveyed speeches. In other words when a senior authority does not point out to the necessity and inevitability of a subject it means that he/she either does not believe in it or the requirements of the speech does not include that subject.

Deeper survey on the authorities' speeches shows that some of them do not clarify on the necessity and inevitability of this subject but other evidences show that they believe in this necessity. For instance, the atmosphere dominating the speeches of Mohammad Hassan Entezari indicates his belief in necessity of using cyberspace although it is not explicitly implied.

On the other hand, some of the conducted emphases (on necessity) are only slogans in a way that they are only stated for rejecting political labels and their arguments are not provided. For instance, Ayatollah Khatami and Ayatollah 
Alam Al-Hoda propose the necessity of using cyberspace but they do not imply its reasons. Some other figures also have not stated the necessity and inevitability of using cyberspace and their attitude is negative and focused on threats; under such conditions lack of belief in necessity of cyberspace is not a very irrational perception.

In general, 4 types of reasoning are proposed by the surveyed figures in favor of necessity and inevitability of using cyberspace:

1- There is no possibility of restricting and eliminating cyberspace and depriving people of using it. This reasoning has been used by the Supreme Leader and commander in chief Ahmadi Moghadam.

2- Cyberspace is a requirement of the current era for promoting religion and politics and using its capacities is a must. This reasoning is usually used by the Supreme Leader, commander in chief Mohammadreza Naghdi and commander in chief Hossein Nejat.

3- Cyberspace is a necessity for the progress of the country, serving people and it's an important leading factor for development. This reasoning is usually used by the Supreme Leader, Dr. Hassan Rouhani, Ali Jannati and Mahmoud Vaezi.

4- Using cyberspace and internet is one of the citizenship rights and also a communication right. This reasoning has been used by Dr. Hassan Rouhani and Mahmoud Vaezi.

\subsection{Position and role of powerful figures in cyberspace}

Based on two-dimensional power model, media is a tool in the hand of powerful group of the society by which they select their own messages. In this model, media discuss the news and reports in favor of that powerful group. Thus, one of the important issues regarding the analysis of political figures' approach toward cyberspace is their attitude toward the position and role of owners and operators of communicational tools and media.

The issue of position and role of powerful figures in cyberspace is reflected in speeches of 18 figures. This issue is one of the most important issues discussed by political figures and authorities and it forms a significant part of their speeches. In fact, this issue is one of the most important concerns of the authorities and lack of implying to this issue by Dr. Hassan Rouhani and Mahmoud Vaezi shows lack of paying attention or emphasizing on this issue by these two figures. In such conditions when most political figures point out the important role of global powers in internet and cyberspace management this lack of mentioning indicates a significant meaning in the approach analysis of these two figures.

In general, it could be said that political authorities have pointed out 4 essential issues regarding the position and role of powerful groups toward the cyberspace:

1- Westerns countries pursue a cultural invasion or soft war against the beliefs, values, religion and morals of a community through cyberspace and internet. This view has been surveyed by 14 individuals (the Supreme Leader, leaders of mid-day congregational Friday prayers, Ali Jannati, Mohammad Hassan Entezari, Ayatollah Sadegh Amoli Larijani, Hojat Al-Islam Gholam Hossein Mohseni Ejei, Dr. Gholam Ali Hadad Adel, Hojat AlIslam Ahmad Salek and military and police authorities).

2- Western countries monitor the data transferred through cyberspace and they use this information for espionage. This view is surveyed by 7 authorities (Hojat Al-Islam Mohammad Alavi, Hojat Al-Islam Heydar Moslehi, Mohammad Hassan Entezari, Ayatollah Sadegh Amoli Larijani, Hojat Al-Islam Gholam Hossein Mohseni Ejei, Hojat Al-Islam Abd Al Reza Tarazi and commander in chief Esmaeil Ahmadi Moghadam).

3- Cyberspace is a tool serving the western countries for overthrowing and changing the independent governments in the world. This view is surveyed by 6 authorities (The Supreme Leader, Ayatollah Ahmad Alam Al-Hoda, Hojat Al-Islam Heydar Moslehi, Hojat Al-Islam Tarazi, Hojat Al-Islam Ahmad Salek, and commander in chief Ahmadi Moghadam).

4- Western countries dominate the cyberspace and have occupied it. This view is surveyed and proposed by 3 authorities (Ayatollah Ahmad Alam Al-Hoda, Mohammad Hassan Entezari and Rouh Allah Nowbakht).

\subsection{Freedom and power of choice of users in cyberspace}

Based on one-dimensional power model, media is a tool which could be potentially used by all individuals and groups for pointing out their views. In fact, media help the diversity of the society and it increases the individuals' power of choice. Explaining the relationship between political power and the power of networks, Castells emphasizes on this freedom and considers it as one of the primary tools of the cyberspace. Thus, one of the important issues regarding the analysis of political figures' approaches toward the cyberspace is analysis of their approaches toward cyberspace users' freedom 
and power of choice.

In comments of 11 political figures some issues are proposed that could be relatively related to the issue of cyberspace users' freedom and power of choice. Lack of pointing out this by the figures either means that they do not believe in freedom and power of choice of users or it is caused by the sampling problems and requirements of the analyzed speeches. In other words, when a political figure does not refer to freedom and power of choice in speeches it means that they either do not believe in that or the requirements of the speech do not include this subject. But deeper survey of the comments of these 9 figures shows that they either do not believe in the freedom and power of choice of users or they are against it.

In general, it could be said that political figures have adopted three different approaches regarding the cyberspace users' freedom and power of choice:

1- Some of the authorities have openly stated their opposition against users' freedom and they have considered it to be traumatic. This group of authorities has inexplicitly accepted the possibility of freedom and power of choice by users in cyberspace but they believe that this subject has negative outcomes and cultural experts must try to stand against it. This view has been discussed by 4 surveyed authorities (Ayatollah Ahmad Khatami, Ayatollah Ahmad Alam Al-Hoda, Ayatollah Sadegh Amoli Larijani and Hojat Al-Islam Gholam Hossein Mohseni Ejei).

2- The second approach is visible among authorities (that despite their negative attitude toward cyberspace) who limitedly emphasize on the necessity of education, civilization and self-protection; thus, they have a slight difference compared to the first approach and they inexplicitly approve the users' power of choice. This view is proposed by 4 authorities (Hojat Al-Islam Mohammad Alavi, Hojat Al-Islam Abd Al-Reza Tarazi, Hojat Al-Islam Ahmad Salek and commander in chief Hossein Nejat). It must be noted that this approach is also followed up by Mahmoud Vaezi (whose view is generally categorized in the third category).

3- Among the surveyed authorities, only three individuals have explicitly accepted and approved the users' freedom and power of choice. Members of the $11^{\text {th }}$ government which means Dr. Hassan Rouhani, Ali Jannati and Mahmoud Vaezi believe that the era of one-way messages is ended and there is no possibility of restricting users' freedom.

\subsection{The most important threats and concerns regarding the cyberspace}

One of the important issues regarding the analysis of authorities' approach toward the cyberspace is that what are the concerns of these individuals toward this new phenomenon? And that what aspects of this phenomenon are considered to be a threat? Surveying this issue is very necessary because it gives the researcher a view regarding the attitude of application of political power toward the cyberspace in the framework of two-dimensional power proposed by Lukes.

In the comments of 19 political authorities some issues are discussed that indicate their concerns regarding cyberspace and internet. In general, it could be said that 8 types of concerns are proposed by political authorities and some of them are also related to each other:

1- Lies and incorrect information

2- Propaganda against the Islamic beliefs and spreading doubts

3- Invasion against cultural and family values

4- Promoting corruption and immoral actionsss

5- Lack of security and abusing information and espionage

6- Western tools toward overthrowing and changing governments

7- Political propaganda against the system and its political figures

8- Internet and cyber crimes

\subsection{The most important opportunities and benefits of cyberspace}

One of the important issues regarding analysis of senior authorities' approach toward cyberspace is that in their perspective what are the opportunities of cyberspace and internet for the society and in other words, what are their benefits. The importance of this attitude is more perceived when we pay attention to this issue that if authorities consider cyberspace as an opportunity rather than a threat then they are less strict toward the cyberspace while making decisions and they reject making decisions about filtering and they will have more interaction with the users.

In comments of 12 senior authorities the opportunities and benefits of internet and cyberspace are discussed. In general, it could be said that in comments of political figures, 3 types of opportunities and benefits are discussed: 
1- Possibility of using it as a propaganda against the enemies

2- Possibility of using it for advertising Islam and revolution in the world

3- Possibility of using it toward development and progress of the country

The authorities' attitude toward cyberspace benefits and opportunities is very general and is not clearly defined; on the other hand, it is at a minimum level and it is based on an ideological intellectual order and they pay less attention to the technological aspects and the interactional capacities in the cyberspace.

\subsection{Efficiency and necessity of filtering in cyberspace}

Filtering in cyberspace is one of the most controversial political and social arguments in the country that has a close relationship with attitude toward cyberspace and internet. Based on two-dimensional power model, cyberspace is not only an equal communicational opportunity and the logic of this model is accompanied by the political power plus filtering, censorship or control. On the other hand, if we deal with internet and cyberspace based on the one-dimensional power then filtering is significantly meaningless and inefficient.

10 political figures have stated the filtering in their speeches. In this regard two main approaches are observed: filtering supporters and filtering opponents. Among the surveyed figures, the Supreme Leader, Ayatollah Ahmad Khatami, Ayatollah Ahmad Alam Al-Hoda, Ayatollah Sadgh Amoli Larijani, Hojat Al-Islam Gholam Hossein Mohseni Ejei and Hojat Al-Islam Abd Al-Reza Tarazi (Supreme Leader, leaders of mid-day congregational Friday prayers, authorities of the Judicial branch) have explicitly supported filtering. Metaphors such as separating thorn from flowers, poisonous syrup and comparing freedom of internet with freedom of drugs are used by this group of political figures.

On the other hand, there are authorities of the $11^{\text {th }}$ government, the president, minister of culture and minister of communications that talk about the uncontrollability of the internet and the harm of this policy and believe that the main solution of the harms existing in the cyberspace is culture promotion.

\subsection{Features of a desirable cyberspace policy}

Surveying the comments of political figures regarding the cyberspace and internet shows that a kind of desirable political approach is pursued in dealing with it. In fact, all politicians have provided recommendations or orders regarding dealing with phenomenon based on their analysis of inevitability and necessity, position and role of the powerful people, users' freedom and power of choice, opportunities and threats of the cyberspace.

In general, it could be said that 20 political figures have proposed 10 types of policies for dealing with cyberspace. It must be noted that some of these policies interfere each other and/or have a relationship with each other. On the other hand, some of the proposed issues are general and they have no clear example; thus, they are not included in this analysis.

1- Training the audience, culture promotion and promoting awareness

2- Filtering, blocking and clearing

3- Localizing the communication infrastructure of cyberspace

4- Judicial approach toward offenders

5- Producing Islamic and revolutionary content

6- Increasing the presence of faithful and revolutionary forces

7- Protecting the privacy

8- Informational and security approach toward the enemy in cyberspace

9- Using this tool for advertising and increasing the effect

10- Using it toward development and progress of the country

\section{Conclusion}

Surveying the views of 20 political figures regarding the cyberspace and internet indicates that there are 4 main approaches regarding dealing with this phenomenon. Naturally it is inevitable to provide general categorization about views without considering some minor differences; but providing this categorization brings about important analysis results for this research. These 4 approaches include:

1- Quite pessimistic approach: this approach is explicitly against cyberspace and internet. It introduces cyberspace as a set of threats and considers its opportunities to be very little. This type of people do not accept the necessity and inevitability of using cyberspace and consider it as a poisonous syrup that although a 
very little part of it is poisonous, drinking it is equal to death. Naturally, this approach does not pay attention to the role of people's awareness while dealing with the harms of cyberspace, and people demands and needs and it considers filtering as the best solution for dealing with this phenomenon.

2- Separating thorn from flower approach: This is a very common approach among the authorities of the country and it does not explicitly resist against cyberspace. The supporters of this approach do not declare their resistance against cyberspace and prefer refinement and separating thorn from flower. In this approach a special emphasis is on existence of filtering and censorship in other countries. This approach has some similarities with the first approach and especially it emphasizes on threats of the cyberspace (and not its opportunities) but it tries to hide the negative and passive attitude.

3- Double-edged knife: the main idea of this approach is balance between opportunities and threats in cyberspace and the method using this is highly important. Supporters of this approach consider cyberspace and internet as an undeniable reality and they declare their explicit approach against the negative and passive attitude. In this approach also the necessity of opponents' awareness and political propaganda are emphasized. This approach is mostly visible among the non-authorities of cyberspace policy making.

4- Optimistic approach: supporters of this approach consider cyberspace as a special feature having more opportunities compared to threats. This approach has a special emphasis on this issue that filtering is inefficient and quarantining the society is impossible. The supporters of this approach also pay attention to the necessity of filtering in some cases that are examples of obscene, espionage and anti-religious contents; but they pursue the intelligent filtering and are against the general filtering and they also consider it a political issue. Education and culture promotion are emphasized in this approach and are considered as an alternative solution in public space.

Surveying different aspects of political power in cyberspace and also after explaining the features and general specification of the research framework, the researcher has extracted indicators in a conceptual model from Lukes's theories that could be a base for analyzing data achieved from qualitative content analysis of the population. Power application methods in cyberspace by the government, desirable policy features of the cyberspace, filtering or antifiltering, attitude toward users' freedom in cyberspace, attitude of authorities toward opportunities and threats and government's efforts for promoting and enriching indicators and components were all based on Lukes's theory of threedimensional power.

Regarding the content analysis of comments of the authorities on cyberspace and its application it could be concluded that most approaches are pessimistic toward the cyberspace and only one approach is optimistic. Thus ,the political system in Iran tends toward having an influence and direct control over the cyberspace and it approves filtering and content control. In this regard in the framework of Lukes's categorization, the official approach of political power in Iran tends toward the two-dimensional approach and right now this approach is being pursued. Also from the perspective of authorities, complete freedom of users in cyberspace is unacceptable and adopting solutions such as censorship and filtering are appreciable.

\section{References}

Davaran, B (2007). Social identity and cybernetic space. Tehran: Culture, art and communication research center. Lukes, S (1996). Power, a radical attitude. Translated by Afrough .E, Tehran: Rasa cultural service institute.

Minavand ,M.GH( 2006). Internet and political development, Public domain in cybernetic space. Journal of political science research. No. 2. Spring and Summer.

Holsti, L.R (1994). Content analysis in social sciences and humanities. Translated by Salarzadeh Amiri .N, Tehran:Allame Tabatabaei University.

Hindes ,B( 2001). Speeches of power (from Hobbes to Foucault). Translated by Younesi .M, Tehran: Shirazeh publications.

Bachrach , Peter \& Baratz, Morton ( 1962 ). The Two Faces of Power .American Political Science Review , 56.

Bachrach , P \& Baratz, M.S ( 1970). Power and Poverty, Theory and Practice. New York : Oxford University Press .

Dahl, Robert ( 1957 ). The Concept of Power. Behavioral Science, 2.

Hsieh, Hsiu-Fang and Sarah E.Shannon (2005 ). Tree Approaches to Qualitative Content Analysis. Qualitative Health Research, November.

Krippendorff , k (2004). Content Analysis: An Introducrion to its Methodology. London: sage .

Lukes ,Steven (2005). Power, A Radical View. Harvard University .

Mayring, Philipp (2000). Qualitative Content Analysis. Qualitative Social Research, Volume 1, No 2 .

Oni (2013) .Open Net Initiative. Retrieved from : http://opennet.net/about-filtering/.

Rader, s (2007). Qualitative Methods of Data Analysis. Zurich: Federal Institute of Technology.

Suler, John (2004).The Psychology of Cyberspace. http://truecenterpoint.com/ce/index.html. 